Research Paper

\title{
Systemic immune-inflammation index (SII) is useful to predict survival outcomes in patients with surgically resected esophageal squamous cell carcinoma
}

\author{
Yibo Gao1, Wei Guo'1, Songhua Cai², Fan Zhang11, Fei Shao', Guochao Zhang1, Tiejun Liu1, Fengwei Tan¹, \\ Ning $\mathrm{Li}^{1}$, Qi Xue ${ }^{1}$, Shugeng Gao ${ }^{1}$, Jie $\mathrm{He}^{1,2}$ \\ 1. Department of Thoracic Surgery, National Cancer Center/National Clinical Research Center for Cancer/Cancer Hospital, Chinese Academy of Medical \\ Sciences and Peking Union Medical College, Beijing 100021, China \\ 2. Department of Thoracic Surgery, National Cancer Center/National Clinical Research Center for Cancer/Cancer Hospital \& Shenzhen Hospital, Chinese \\ Academy of Medical Sciences and Peking Union Medical College, Shenzhen, 518116, China \\ $\square$ Corresponding author: Dr Jie He, Department of Thoracic Surgery, National Cancer Center/National Clinical Research Center for Cancer/Cancer Hospital, \\ Chinese Academy of Medical Sciences and Peking Union Medical College, Beijing 100021, The People's Republic of China; Email: prof.jiehe@gmail.com \\ ( $)$ Ivyspring International Publisher. This is an open access article distributed under the terms of the Creative Commons Attribution (CC BY-NC) license \\ (https://creativecommons.org/licenses/by-nc/4.0/). See http://ivyspring.com/terms for full terms and conditions.
}

Received: 2018.09.29; Accepted: 2019.04.23; Published: 2019.06.02

\begin{abstract}
Background: The systemic immune-inflammation index (SII) has been reported to be associated with patient survival in various kinds of solid tumors. However, just few studies have focused on its prognostic value in patients with surgically resected esophageal squamous cell carcinoma (ESCC).

Materials and Methods: This study was a single-institution, retrospective analysis of 468 ESCC patients who underwent curative esophagectomy at the Department of Thoracic Surgery, National Cancer Center/Cancer Hospital, Chinese Academy of Medical Sciences and Peking Union Medical College between 2005 and 2008. The receiver operating curve (ROC) was plotted to compare the discrimination ability of the SII and other inflammatory factors for overall survival (OS) and disease-free survival (DFS). Univariate and multivariate analyses were performed based on the Cox proportional hazards regression model.
\end{abstract}

Results: The SII, neutrophil-lymphocyte ratio (NLR), and platelet-lymphocyte ratio (PLR) were all associated with OS in ESCC patients. The SII, NLR, and PLR were independent prognostic factors for OS (hazard ratio $(\mathrm{HR})=1.604,95 \%$ confidence interval $(\mathrm{Cl}) 1.247-2.063, \mathrm{P}<0.001 ; \mathrm{HR}=1.396$, $95 \% \mathrm{Cl} 1.074-1.815, \mathrm{P}=0.013 ; \mathrm{HR}=1.370,95 \% \mathrm{Cl} 1.067-1.758, \mathrm{P}=0.013$, respectively) and DFS $(\mathrm{HR}=1.681,95 \% \mathrm{Cl} 1.307-2.162, \mathrm{P}<0.001 ; \mathrm{HR}=1.376,95 \% \mathrm{Cl} 1.059-1.788, \mathrm{P}=0.017 ; \mathrm{HR}=$ $1.398,95 \% \mathrm{Cl} 1.089-1.794, P=0.009$, respectively). The area under the curve (AUC) for Sll was bigger than NLR, PLR, and MLR (0.553, 0.540, 0.532, and 0.521 , respectively).

Conclusion: The SII is a simple and promising prognostic predictor for patients with surgically resected ESCC. The prognostic value of SII is superior to those of the NLR, PLR and MLR. Moreover, the SIl retained prognostic significance in stage I-II ESCC subgroup (OS, DFS) and stage III ESCC subgroup (DFS).

Key words: esophageal squamous cell carcinoma; systemic immune-inflammation index; neutrophil-to-lymphocyte ratio; platelet-to-lymphocyte ratio; monocyte-to-lymphocyte ratio; prognosis

\section{Introduction}

Esophageal cancer (EC) is currently the fifth most commonly diagnosed malignancy and the fourth leading cause of cancer deaths in China [1]. Esophageal squamous cell carcinoma (ESCC) and esophageal adenocarcinoma (EAC) constitute two major histological types of EC. ESCC is the most common histological type in Chinese patients, accounting for $90 \%$ of all cases, whereas EAC is 
predominant in many western countries [2]. So far, the standard care for ESCC patients is curative esophagectomy, with or without chemoradiation therapy. Unfortunately, ESCC patients still show a poor five-year survival rate of $<30 \%$, even after curative therapy and eventually developing rapid clinical progression $[3,4]$.Therefore, it is imperative to evaluate better prognostic factors, particularly serum predictive indicators, in ESCC patients.

Recent studies have demonstrated that a preoperative inflammatory response could be associated with tumor progression and metastasis and have a significant predictive and prognostic value in various types of cancers [5-8]. There is increasing recognition that the NLR, PLR, and MLR are three markers of systemic inflammation and the elevated values of them might lead to a poor prognosis in various solid tumors, including ESCC [9-12]. However, these three inflammatory factors only integrate two cells. SII, which based on peripheral neutrophil, platelet, and lymphocyte counts, was recently investigated as a novel prognostic marker [13-16]. And to our best knowledge, just few studies regarding SII in patients with surgically resected ESCC are available. Thus, we conducted the present study to investigate and verify the prognostic value of the SII for a larger cohort of patients with surgically resected ESCC.

\section{Materials and Methods}

\section{Patients}

We retrospectively reviewed a database of medical records of 530 consecutive patients with surgically resected ESCC between 2005 and 2008 at the department of thoracic surgery, National Cancer Center/Cancer Hospital, Chinese Academy of Medical Sciences, and Peking Union Medical College. The inclusion criteria were as follows: (1) curative esophagectomy with R0 resection; (2) histologically confirmed ESCC; 3) preoperative serum laboratory results were obtained within five days before surgery. The exclusion criteria were as follows: (1) if patients received preoperative chemotherapy/radiotherapy; (2) if they had acute/chronic infection or (3) if they had hematological or autoimmune disease; (4) if they died during the perioperative period; (5) if they lacked detailed clinical information and follow-up information. Totally, 62 patients were excluded from this study. The whole enrollment process was clearly shown in Figure 1. Among these patients, 15 patients had infection, hematological or autoimmune disease; 12 patients had incomplete medical data; and 35 patients were lost to follow-up. Finally, a total of 468 patients were enrolled in the present study.

All included subjects provided written informed consent for the study protocol that was approved by the Ethical Committees of the National Cancer Center/Cancer Hospital, Chinese Academy of Medical Sciences, and Peking Union Medical College. Patients were followed up in the outpatient department every 3-6 months for the first two years after surgery and then annually. The follow-up included recording the medical history, physical examinations, chest computed tomography, and endoscopy (if necessary). The last follow-up was on July $9^{\text {th }} 2015$. And in our study, the primary endpoints were 5-year OS and 5-year DFS.

\section{Clinicopathological parameters}

The clinicopathological parameters of the patients, including age, gender, smoking history, drinking history, tumor location, tumor differentiation grade, maximum tumor diameter, lymph node metastasis, TNM stage, operation time, and intraoperative blood loss, were obtained from the medical records. The pathological classification of the primary tumor, degree of lymph node metastasis, and presence of distant metastasis were assessed based on the American Joint Committee on Cancer staging manual (seventh edition) [17].

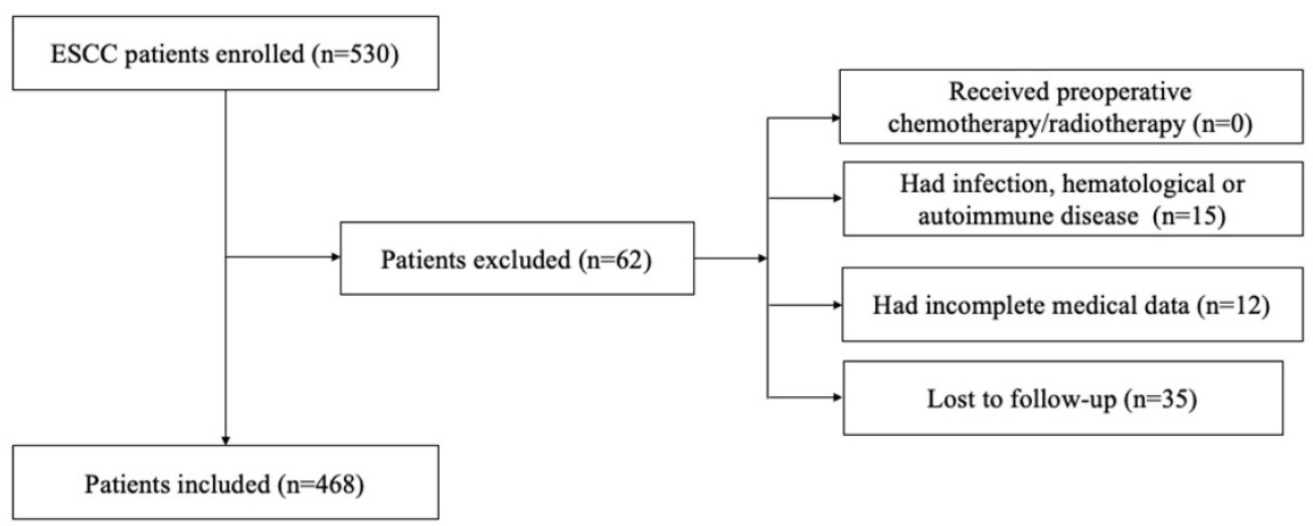

Figure 1. The flowchart of the enrollment process 


\section{Blood sample analysis}

The laboratory data on preoperative CBC counts were retrospectively extracted from medical records. All these results were obtained within five days before surgery. $\mathrm{CBC}$ was measured using EDTA-treated blood and analyzed using an automated hematology analyzer XE-2100 (SYSMEX hematology analyzer; Medical Electronics, Kobe, Japan).

\section{SII, NLR, PLR, and LMR evaluations}

Data on preoperative blood cell counts were retrospectively extracted from the medical records. White blood cell count data were analyzed in the general routine laboratory of our hospital within one week before surgery. We calculated the SII, NLR, PLR and MLR as follows: SII $=$ platelet counts $\times$ neutrophil counts/lymphocyte counts, NLR $=$ neutrophil counts/lymphocyte counts, PLR $=$ platelet counts/lymphocyte counts, MLR = monocyte counts/lymphocyte counts.

\section{Statistical analysis}

We used SPSS version 19.0 (IBM Corp., Armonk, NY, USA) to perform all statistical analyses. The Pearson Chi-square test was used to compare categorical variables. Multiple linear regression analyses were used to determine factors associated with SII, NLR, PLR, and MLR. We constructed the Receiver operated characteristics (ROC) curves to determine the cut-off values for SII, NLR, PLR, and MLR that yielded the joint maximum sensitivity and specificity. The Kaplan-Meier method was used for univariate analysis, and the difference between groups was assessed by the log-rank test. The Cox proportional hazard model was used for multivariate analysis, and hazard ratios (HRs) with 95\% confidence intervals (CIs) were used to quantify the strength of the association between predictors and survival. A two-sided value of $\mathrm{P}<0.05$ was considered statistically significant.

\section{Results}

\section{Patients characteristics}

The 468 patients in this study were $376(80.3 \%)$ men and 92 (19.7\%) women (Table 1). The median age was 59.5 years (range, 36-81 years). The distribution of pathological stages was as follows: stage I, 46 (9.8\%); stage II, 199 (42.6\%); and stage III, 223 (47.6\%). The mean follow-up duration was $49.1 \pm 32.6$ months (range, 3.2-114.5 months). At the end of the last follow-up, $270(57.7 \%)$ patients had died.

The median preoperative SII, NLR, PLR, and MLR were 374.26 (range, 53.28-4748.19), 1.98 (range,
$0.52-11.00$ ), 109.66 (range, 32.79-418.52), and 0.148 (range, 0.003-0.854), respectively.

Table 1. The characteristics of the 468 patients grouped by SIl

\begin{tabular}{|c|c|c|c|c|}
\hline & Cases (number, \%) & SII (num & ber) & $P$ value \\
\hline & $468(100)$ & $<479.72$ & $\geq 479.72$ & \\
\hline Gender & & & & $0.005^{*}$ \\
\hline Male & $376(80.3)$ & 250 & 126 & \\
\hline Female & $92(19.7)$ & 75 & 17 & \\
\hline Age (years) & & & & 0.484 \\
\hline$\leq 60$ & $247(52.8)$ & 168 & 79 & \\
\hline$>60$ & $221(47.2)$ & 157 & 64 & \\
\hline Smoking & & & & 0.546 \\
\hline Ever & 209 (44.7) & 142 & 67 & \\
\hline Never & $259(55.3)$ & 183 & 76 & \\
\hline Drinking & & & & 0.309 \\
\hline Yes & $286(61.1)$ & 189 & 97 & \\
\hline No & $182(38.9)$ & 136 & 46 & \\
\hline Tumor length & & & & 0.261 \\
\hline$\leq 3$ & $70(15)$ & 53 & 17 & \\
\hline$>3$ & $398(85)$ & 272 & 126 & \\
\hline Tumor location & & & & 0.455 \\
\hline Upper & $62(13.2)$ & 47 & 15 & \\
\hline Middle & $228(48.7)$ & 154 & 74 & \\
\hline Lower & $178(38.0)$ & 124 & 54 & \\
\hline Differentiation & & & & 0.841 \\
\hline Well & $123(26.3)$ & 88 & 35 & \\
\hline Moderate & $233(49.8)$ & 160 & 73 & \\
\hline Poor & $112(23.9)$ & 77 & 35 & \\
\hline T stage & & & & 0.147 \\
\hline $\mathrm{T} 1$ & $46(9.8)$ & 38 & 8 & \\
\hline $\mathrm{T} 2$ & $77(16.5)$ & 51 & 26 & \\
\hline $\mathrm{T} 3$ & $274(58.5)$ & 191 & 83 & \\
\hline $\mathrm{T} 4$ & $71(15.2)$ & 45 & 26 & \\
\hline N stage & & & & 0.287 \\
\hline No & $238(50.9)$ & 166 & 72 & \\
\hline N1 & $129(27.6)$ & 91 & 38 & \\
\hline $\mathrm{N} 2$ & $74(15.8)$ & 46 & 28 & \\
\hline N3 & $27(5.8)$ & 22 & 5 & \\
\hline TNM stage & & & & 0.169 \\
\hline I & $46(9.8)$ & 37 & 9 & \\
\hline II & $199(42.5)$ & 132 & 67 & \\
\hline III & $223(47.6)$ & 156 & 67 & \\
\hline Operation time (min) & & & & 0.312 \\
\hline$<200$ & $200(42.9)$ & 144 & 56 & \\
\hline$\geq 200$ & $268(57.1)$ & 181 & 87 & \\
\hline Intraoperative blood loss (ml) & & & & $0.044^{*}$ \\
\hline$<200$ & $220(47.0)$ & 163 & 47 & \\
\hline$\geq 200$ & $248(53.0)$ & 162 & 86 & \\
\hline
\end{tabular}

${ }^{*} P$ less than 0.05 is significant.

$\mathrm{SII}=$ systemic immune-inflammation index

\section{Selection of optimal cut-off values for SII, NLR, PLR and MLR}

As shown in Figure 2, the AUC was 0.553, 0.540, 0.532, and 0.521 for SII, NLR, PLR, and MLR, respectively. The optimal cut-off values for the prediction of survival were 479.72 for SII, 2.27 for NLR, 117.07 for PLR and 0.19 for MLR. Consequently, patients were separately divided into two groups with high or low levels according to the optimal cut-off values. One hundred and forty-three patients $(30.6 \%)$ had SII $\geq 479.72,127$ patients $(27.1 \%)$ had NLR $\geq 2.27,153$ patients $(32.7 \%)$ had PLR $\geq 117.07$ and 165 $(35.3 \%)$ patients had MLR $\geq 0.19$. 


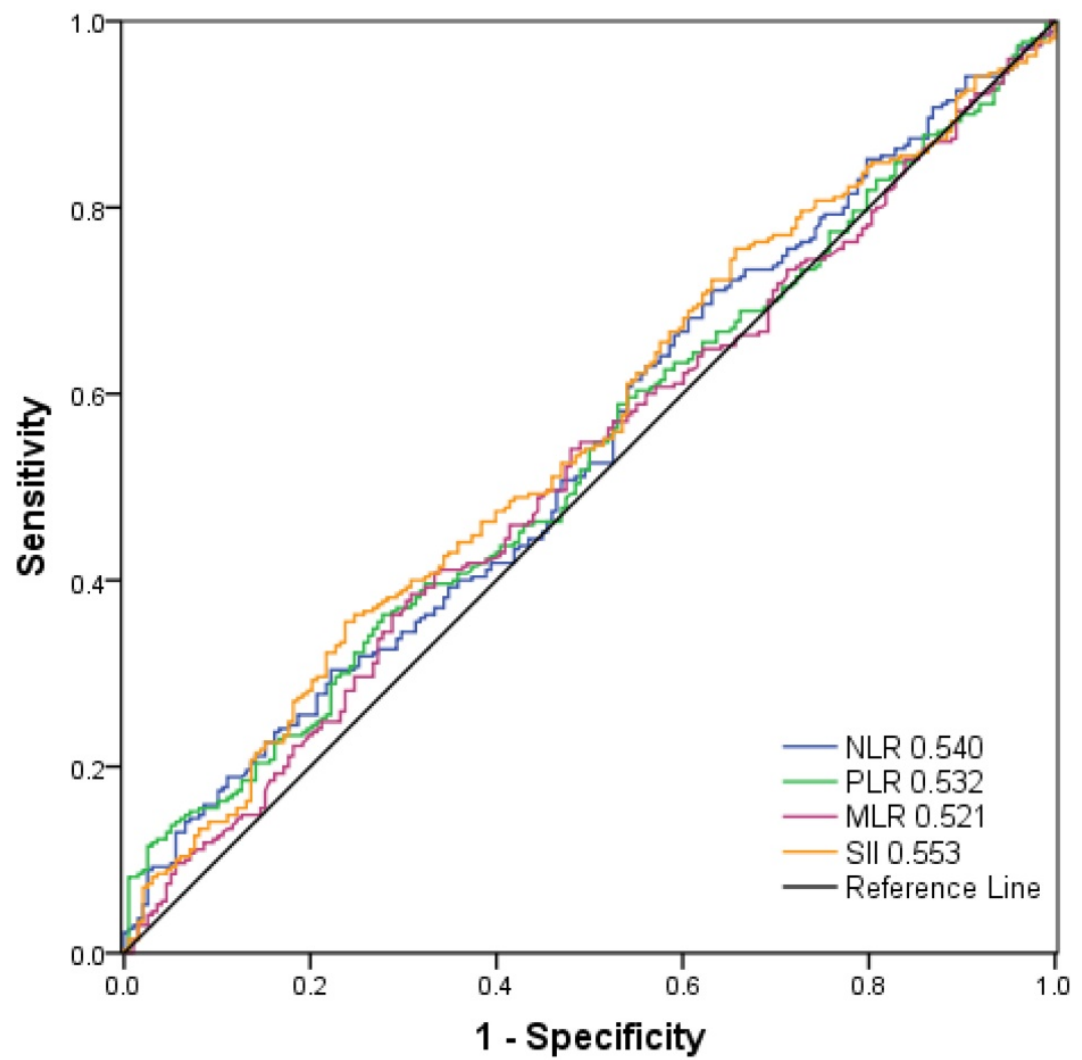

Figure 2. Receiver operating characteristic curve analysis for the optimal cut-off value of SII, NLR, PLR and MLR. The areas under the curve for overall survival were 0.553 , $0.540,0.532$, and 0.521 for SII, NLR, PLR and MLR, respectively.

\section{Correlation between the clinicopathological parameters and SII, NLR, PLR, and MLR}

The relationship between the clinicopathological parameters and SII is shown in Table 1. The preoperative SII were associated with gender $(\mathrm{p}=$ $0.005)$ and more intraoperative blood loss $(p=0.044)$ (Table 1).

The relationship between the clinicopathological parameters and NLR, PLR, and MLR is shown in Table 2 . The preoperative NLR were associated with gender $(p=0.005)$, tumor length $(p=0.020)$, and $T$ stage $(p=0.010)$. The preoperative MLR were associated with tumor length $(\mathrm{p}=0.010)$ and $\mathrm{T}$ stage $(\mathrm{p}=0.024)$ (Table 2$)$.

\section{The prognostic value of SII, NLR, PLR, and MLR}

OS curves were plotted by the Kaplan-Meier method and compared using the log-rank test. High SII, NLR, PLR and MLR were associated with poor OS ( $p=0.001, p=0.033$, and $p=0.027$, respectively) (Figure 3A-3C) and poor DFS $(\mathrm{p}<0.001, \mathrm{p}=0.034$, and $p=0.024$, respectively) (Figure 4A-4C). However, no significant differences were found between MLR and patients' OS $(\mathrm{p}=0.104)$ (Figure 3D) and DFS $(\mathrm{p}=$ 0.093) (Figure 4D).
The prognostic value of SII was next investigated in stage I/II and stage III subgroup, separately. As shown in Figure 5 and Figure 6, patients with SII $\geq$ 479.72 have worse OS in stage I/II subgroup ( $p=$ 0.002 ) (Figure 5A), but have no significant effects in stage III subgroup $(p=0.058)$ (Figure $6 \mathrm{~A})$. As for DFS, patients with SII $\geq 479.72$ have worse DFS in stage I/II subgroup $(\mathrm{p}=0.001)$ (Figure $5 \mathrm{~B})$ and stage III subgroup $(p=0.024)$ (Figure 6B).

\section{Univariate and multivariate analyses}

Univariate analyses demonstrated that age, smoking history, $\mathrm{T}$ stage, lymph node metastasis, TNM stage, operation time, intraoperative blood loss, SII, NLR, PLR were significant risk factors for a poor prognosis for OS and DFS (Table 3). To avoid multicollinearity, we conducted the multivariate analysis using four models separately. Each multivariate model included only one immune-inflammatory indicator (SII, NLR, PLR, or MLR). The results revealed age $(\mathrm{P}<0.001)$, TNM stage $(\mathrm{P}<0.001)$, operation time $(\mathrm{P}=0.030)$, high SII levels $(\mathrm{P}=0.001)$, high NLR $(\mathrm{P}=0.013)$ and high PLR $(\mathrm{P}=$ $0.013)$ were found to be independently associated with a poor OS or DFS in the multivariate analysis (Table 4). 

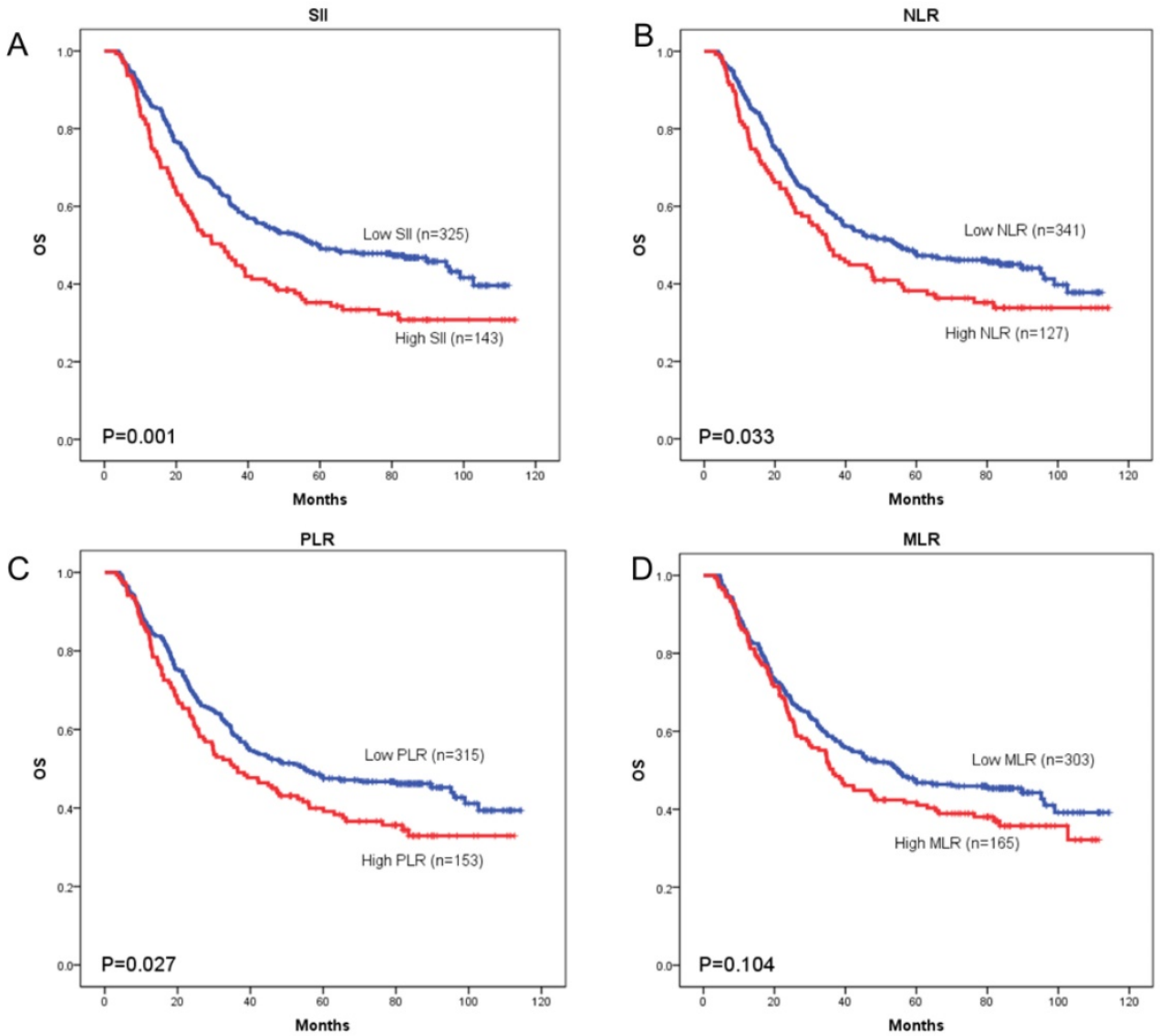

Figure 3. Kaplan-Meier curves of overall survival (OS) according to SII (A), NLR (B), PLR (C) and MLR (D)

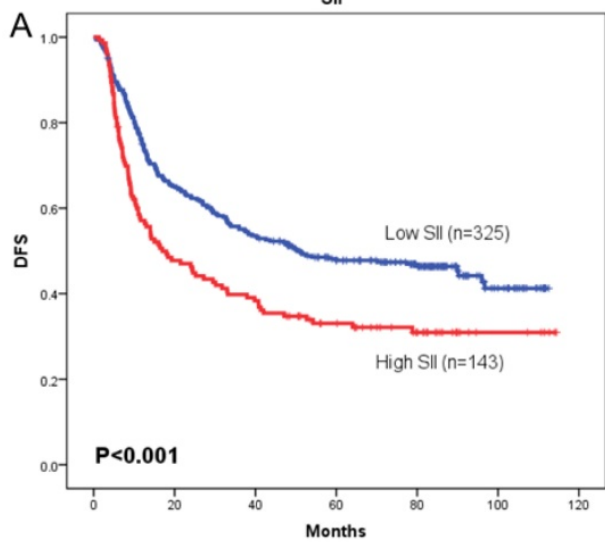

PLR

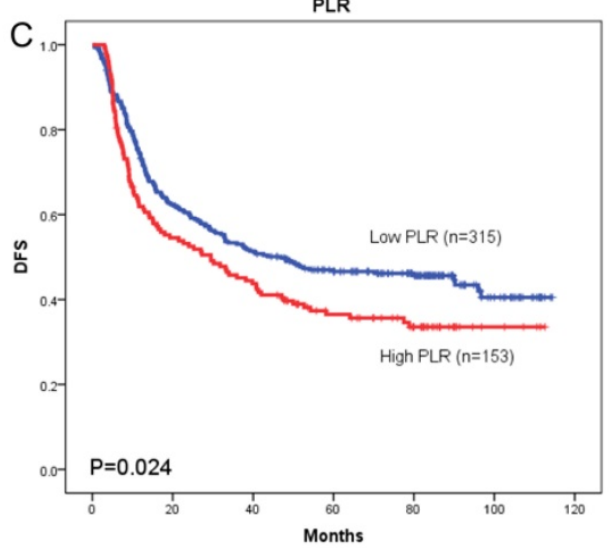

NLR

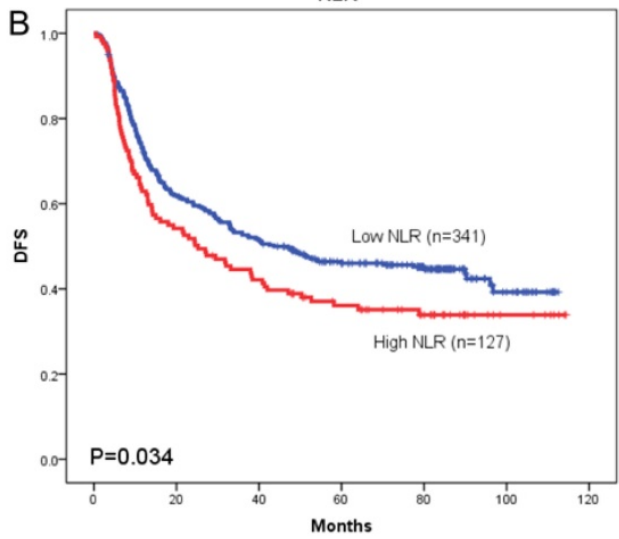

MLR

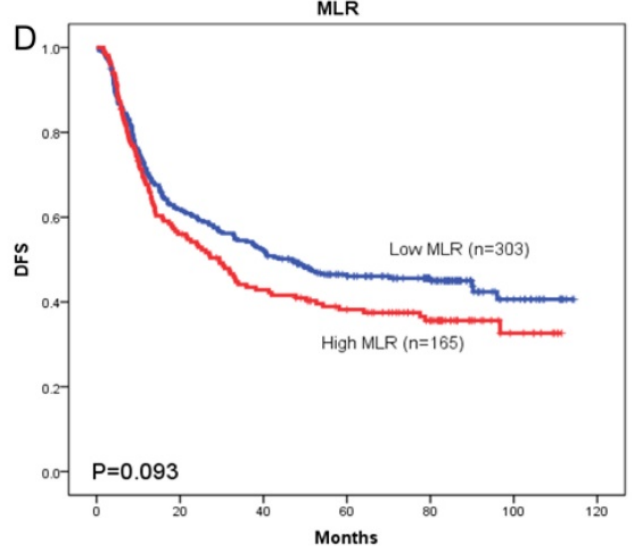

Figure 4. Kaplan-Meier curves of disease-free survival (DFS) according to SII (A), NLR (B), PLR (C) and MLR (D) 
Table 2. The characteristics of the 468 patients grouped by NLR, PLR and MLR

\begin{tabular}{|c|c|c|c|c|c|c|c|c|c|c|}
\hline & \multirow{2}{*}{$\begin{array}{l}\text { Cases (number, \%) } \\
468(100)\end{array}$} & \multicolumn{2}{|c|}{ NLR (number) } & \multirow[t]{2}{*}{$P$ value } & \multicolumn{2}{|c|}{ PLR (number) } & \multirow[t]{2}{*}{$P$ value } & \multicolumn{2}{|c|}{ MLR (number) } & \multirow[t]{2}{*}{$P$ value } \\
\hline & & $<2.27$ & $\geq 2.27$ & & $<117.05$ & $\geq 117.05$ & & $<0.19$ & $\geq 0.19$ & \\
\hline Gender & & & & $0.005^{*}$ & & & 0.172 & & & 0.223 \\
\hline Male & $376(80.3)$ & 266 & 126 & & 259 & 117 & & 238 & 138 & \\
\hline Female & $92(19.7)$ & 75 & 17 & & 56 & 36 & & 65 & 27 & \\
\hline Age (years) & & & & 0.349 & & & 0.490 & & & 0.562 \\
\hline$\leq 60$ & $247(52.8)$ & 175 & 72 & & 170 & 77 & & 163 & 84 & \\
\hline$>60$ & $221(47.2)$ & 166 & 55 & & 145 & 76 & & 140 & 81 & \\
\hline Smoking & & & & 0.917 & & & 0.428 & & & 0.560 \\
\hline Ever & $209(44.7)$ & 153 & 56 & & 145 & 64 & & 132 & 77 & \\
\hline Never & 259 (55.3) & 188 & 71 & & 170 & 89 & & 171 & 88 & \\
\hline Drinking & & & & 0.287 & & & 0.615 & & & 0.921 \\
\hline Yes & $286(61.1)$ & 203 & 83 & & 195 & 91 & & 186 & 100 & \\
\hline No & $182(38.9)$ & 138 & 44 & & 120 & 62 & & 117 & 65 & \\
\hline Tumor length & & & & $0.020^{*}$ & & & 0.128 & & & $0.010^{*}$ \\
\hline$\leq 3$ & $70(15)$ & 59 & 11 & & 53 & 17 & & 55 & 15 & \\
\hline$>3$ & $398(85)$ & 282 & 116 & & 262 & 136 & & 248 & 150 & \\
\hline Tumor location & & & & 0.670 & & & 0.922 & & & 0.670 \\
\hline Upper & $62(13.2)$ & 47 & 15 & & 43 & 19 & & 47 & 15 & \\
\hline Middle & $228(48.7)$ & 162 & 66 & & 152 & 76 & & 162 & 66 & \\
\hline Lower & $178(38.0)$ & 132 & 46 & & 120 & 58 & & 132 & 46 & \\
\hline Differentiation & & & & 0.274 & & & 0.972 & & & 0.260 \\
\hline Well & $123(26.3)$ & 92 & 31 & & 82 & 41 & & 87 & 36 & \\
\hline Moderate & $233(49.8)$ & 174 & 59 & & 158 & 75 & & 147 & 86 & \\
\hline Poor & $112(23.9)$ & 75 & 37 & & 75 & 37 & & 69 & 43 & \\
\hline T stage & & & & $0.010^{*}$ & & & 0.727 & & & $0.024^{*}$ \\
\hline $\mathrm{T} 1$ & $46(9.8)$ & 41 & 5 & & 32 & 14 & & 34 & 12 & \\
\hline $\mathrm{T} 2$ & 77 (16.5) & 49 & 28 & & 54 & 23 & & 47 & 30 & \\
\hline T3 & $274(58.5)$ & 204 & 70 & & 185 & 89 & & 186 & 88 & \\
\hline $\mathrm{T} 4$ & 71 (15.2) & 47 & 24 & & 44 & 27 & & 36 & 35 & \\
\hline $\mathrm{N}$ stage & & & & 0.988 & & & 0.634 & & & 0.398 \\
\hline No & 238 (50.9) & 173 & 65 & & 156 & 82 & & 158 & 80 & \\
\hline N1 & $129(27.6)$ & 95 & 34 & & 91 & 38 & & 86 & 43 & \\
\hline N2 & $74(15.8)$ & 54 & 20 & & 48 & 26 & & 45 & 29 & \\
\hline N3 & $27(5.8)$ & 19 & 8 & & 20 & 7 & & 14 & 13 & \\
\hline TNM stage & & & & 0.204 & & & 0.278 & & & 0.228 \\
\hline I & $46(9.8)$ & 38 & 8 & & 33 & 13 & & 35 & 11 & \\
\hline II & $199(42.5)$ & 139 & 60 & & 126 & 73 & & 125 & 74 & \\
\hline III & $223(47.6)$ & 164 & 59 & & 156 & 67 & & 143 & 80 & \\
\hline Operation time (min) & & & & 0.077 & & & 0.163 & & & 0.696 \\
\hline$<200$ & $200(42.9)$ & 153 & 47 & & 142 & 58 & & 132 & 68 & \\
\hline$\geq 200$ & $268(57.1)$ & 188 & 80 & & 173 & 95 & & 171 & 97 & \\
\hline Intraoperative blood loss (ml) & & & & 0.253 & & & 1.000 & & & 0.698 \\
\hline$<200$ & $220(47.0)$ & 166 & 54 & & 148 & 72 & & 140 & 80 & \\
\hline$\geq 200$ & $248(53.0)$ & 175 & 73 & & 167 & 81 & & 163 & 85 & \\
\hline
\end{tabular}

${ }^{*} P$ less than 0.05 is significant.

NLR = neutrophil-lymphocyte ratio; PLR = platelet-lymphocyte ratio; MLR = monocyte-lymphocyte ratio.

Table 3. Univariate analysis with regard to OS in 468 patients with ESCC

\begin{tabular}{|c|c|c|c|c|c|c|}
\hline & OS & & & DFS & & \\
\hline & Pvalue & HR & $95 \% \mathrm{CI}$ & $\mathrm{P}$ value & HR & $95 \% \mathrm{CI}$ \\
\hline Gender (female, male) & 0.589 & 1.088 & $0.800-1.479$ & 0.496 & 1.113 & $0.818-1.513$ \\
\hline Age $(\leq 60,>60)$ & $0.001^{*}$ & 1.517 & $1.192-1.927$ & $0.003^{*}$ & 1.435 & $1.129-1.823$ \\
\hline Smoking (ever, never) & $0.033^{*}$ & 1.297 & $1.021-1.647$ & $0.025^{*}$ & 1.313 & $1.034-1.668$ \\
\hline Drinking (yes, no) & 0.576 & 1.073 & $0.839-1.372$ & 0.602 & 1.068 & $0.835-1.366$ \\
\hline Tumor size $(\mathrm{cm})(\leq 3,>3)$ & 0.139 & 1.307 & $0.917-1.865$ & 0.105 & 1.341 & $0.940-1.913$ \\
\hline Tumor location (upper/middle, lower) & 0.847 & 1.024 & $0.802-1.309$ & 0.981 & 0.997 & $0.780-1.274$ \\
\hline Differentiation (well/moderate, poor) & 0.408 & 1.122 & $0.854-1.473$ & 0.316 & 1.150 & $0.876-1.509$ \\
\hline T stage (T1/T2, T3/T4) & $0.004^{*}$ & 1.525 & $1.143-2.036$ & $0.005^{*}$ & 1.515 & $1.135-2.023$ \\
\hline Lymph node metastasis (negative, positive) & $<0.001^{*}$ & 2.136 & $1.672-2.728$ & $<0.001^{*}$ & 2.117 & $1.658-2.703$ \\
\hline TNM stage (I/II, III) & $<0.001^{*}$ & 2.304 & $1.805-2.941$ & $<0.001^{*}$ & 2.310 & $1.811-2.948$ \\
\hline Operation time $(\mathrm{min})(<200, \geq 200)$ & $0.049^{*}$ & 1.278 & $1.001-1.632$ & $0.026^{*}$ & 1.319 & $1.033-1.684$ \\
\hline Intraoperative blood loss $(\mathrm{ml})(<200, \geq 200)$ & $0.013^{*}$ & 1.357 & $1.066-1.728$ & $0.007^{*}$ & 1.392 & $1.093-1.772$ \\
\hline SII $(<479.72, \geq 479.72)$ & $0.001^{*}$ & 1.521 & $1.185-1.953$ & $<0.001^{*}$ & 1.583 & $1.233-2.032$ \\
\hline $\operatorname{NLR}(<2.27, \geq 2.27)$ & $0.033^{*}$ & 1.325 & $1.022-1.718$ & $0.035^{*}$ & 1.323 & $1.020-1.715$ \\
\hline PLR $(<117.05, \geq 117.05)$ & $0.028^{*}$ & 1.321 & $1.031-1.694$ & $0.024^{*}$ & 1.331 & $1.038-1.706$ \\
\hline $\operatorname{MLR}(<0.19, \geq 0.19)$ & 0.104 & 1.225 & $0.959-1.566$ & 0.094 & 1.234 & $0.965-1.576$ \\
\hline
\end{tabular}

${ }^{*} P$ less than 0.05 is significant.

$\mathrm{CI}=$ confidence interval; $\mathrm{OS}=$ overall survival; $\mathrm{HR}=$ hazard ratio;

$\mathrm{SII}=$ systemic immune-inflammation index; NLR = neutrophil-lymphocyte ratio; PLR = platelet-lymphocyte ratio; MLR = monocyte-lymphocyte ratio 
Table 4. Multivariate analysis with regard to OS in 468 patients with ESCC

\begin{tabular}{|c|c|c|c|c|c|c|}
\hline & OS & & & DFS & & \\
\hline & P value & HR & $95 \% \mathrm{CI}$ & P value & HR & $95 \% \mathrm{CI}$ \\
\hline Age $(\leq 60,>60$ years $)$ & $<0.001^{*}$ & 1.606 & $1.260-2.046$ & $0.001^{*}$ & 1.520 & $1.193-1.937$ \\
\hline Smoking (ever, never) & 0.163 & 1.191 & $0.932-1.523$ & 0.176 & 1.183 & $0.928-1.508$ \\
\hline Differentiation (well/moderate, poor) & 0.522 & 1.094 & $0.831-1.441$ & 0.444 & 1.113 & $0.846-1.464$ \\
\hline $\mathrm{T}$ stage $(\mathrm{T} 1 / \mathrm{T} 2, \mathrm{~T} 3 / \mathrm{T} 4)$ & 0.761 & 1.055 & $0.746-1.494$ & 0.801 & 1.046 & $0.739-1.480$ \\
\hline Lymph node metastasis (negative, positive) & 0.221 & 1.295 & $0.856-1.957$ & 0.276 & 1.258 & $0.832-1.901$ \\
\hline TNM stage (I/II, III) & $<0.001^{*}$ & 2.330 & $1.825-2.975$ & $<0.001^{*}$ & 2.355 & $1.845-3.006$ \\
\hline Operation time $(\mathrm{min})(<200, \geq 200)$ & $0.030^{*}$ & 1.314 & $1.027-1.682$ & $0.012^{*}$ & 1.372 & $1.307-2.162$ \\
\hline Intraoperative blood loss $(\mathrm{ml})(<200, \geq 200)$ & 0.231 & 1.166 & $0.907-1.498$ & 0.180 & 1.186 & $0.924-2.105$ \\
\hline SII $(<479.72, \geq 479.72)$ & $<0.001^{*}$ & 1.604 & $1.247-2.063$ & $<0.001^{*}$ & 1.681 & $1.307-2.162$ \\
\hline $\operatorname{NLR}(<2.27, \geq 2.27)$ & $0.013^{*}$ & 1.396 & $1.074-1.815$ & $0.017^{*}$ & 1.376 & $1.059-1.788$ \\
\hline PLR $(<117.05, \geq 117.05)$ & $0.013^{*}$ & 1.370 & $1.067-1.758$ & $0.009^{*}$ & 1.398 & $1.089-1.794$ \\
\hline $\operatorname{MLR}(<0.19, \geq 0.19)$ & 0.173 & 1. 188 & $0.927-1.522$ & 0.118 & 0.820 & $0.640-1.052$ \\
\hline
\end{tabular}

${ }^{*} P$ less than 0.05 is significant.

$\mathrm{CI}=$ confidence interval; $\mathrm{OS}=$ overall survival; $\mathrm{HR}=$ hazard ratio;

$\mathrm{SII}=$ systemic immune-inflammation index; NLR = neutrophil-lymphocyte ratio; PLR = platelet-lymphocyte ratio; MLR = monocyte-lymphocyte ratio
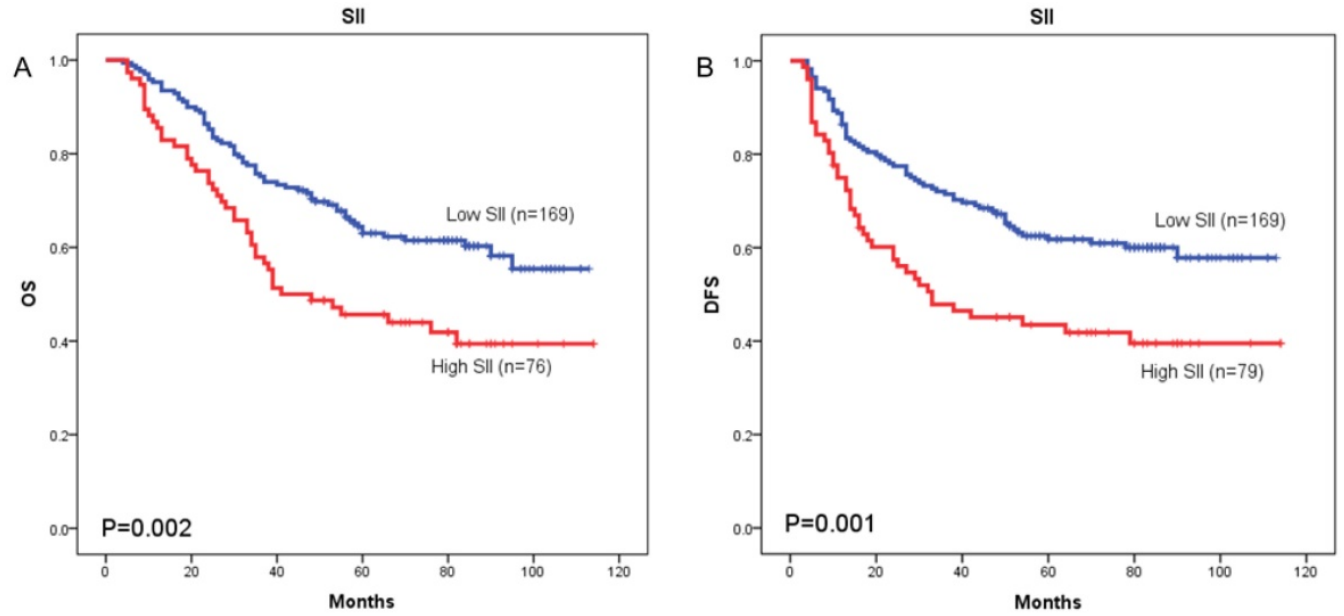

Figure 5. Kaplan-Meier OS curves (A) and DFS curves (B) for patients with TNM stage I-II disease
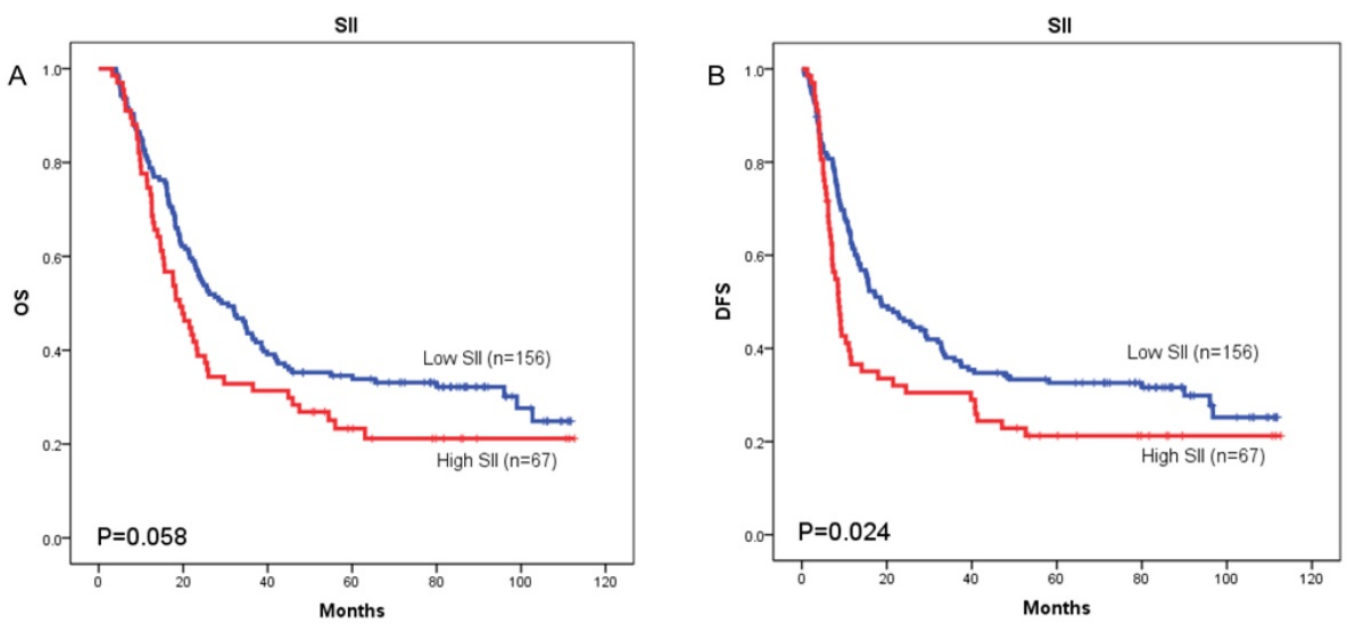

Figure 6. Kaplan-Meier OS curves (A) and DFS curves (B) for patients with TNM stage III disease

\section{Discussion}

In recent years, increasing evidence demonstrated that there is a strong association between systemic inflammation and cancer [18-20]. In the present study, we retrospectively analyzed a consecutive cohort of 468 ESCC patients who underwent curative esophagectomy with $\mathrm{R} 0$ resection in our center. To our best knowledge, the population size and the quality of follow-up of the present study were much better than other two similar studies [21, 22]. It was worth mentioning that our study focused on the prognostic value of the SII for both OS and DFS of the ESCC patients. Our result showed that a high SII was an independent predictor for both OS and DFS in patients with surgically resected ESCC, and by 
comparing the areas under the AUC curve, SII was superior to NLR, PLR, and MLR in terms of prognostic ability. We also found that high SII was associated with gender and intraoperative blood loss. Moreover, we divided the 468 patients into two different groups according to their TNM stage for further analysis. The subgroup results showed that SII retained prognostic significance in stage I-II ESCC subgroup (OS, DFS) and stage III ESCC subgroup (DFS).

Systemic inflammatory factors such as NLR, PLR and MLR have been found to be independent markers of prognosis in a variety of cancers, including ESCC [23-27]. Feng [24] et al. revealed that preoperative NLR and PLR were significant predictors of OS in patients with ESCC and that PLR was superior to NLR as a prognostic index. Nakamura et al. [25] and Yutong et al. [26] have suggested that an increased NLR is associated with tumor progression and poor survival in EC patients. Recently, a meta-analysis by Zhao et al. [28] showed that a higher PLR might be a significant predictive biomarker for EC patients. Hirahara et al. $[23,27]$ also demonstrated that a decreased LMR was an independent prognostic factor for OS in ESCC patients. SII, based on neutrophil, platelet, and lymphocyte counts, was shown to be an independent prognostic indicator for hepatocellular carcinoma, lung cancer, colorectal cancer, renal cancer, prostate cancer and gastric cancer patients [12, 13, 15, 29-31].

The mechanism by which high SII contributes to a poor prognosis in patients with solid cancer is still unclear. Several potential theories may be used to explain the prognostic values of SII. Firstly, neutrophils expand both in the tumor microenvironment and systemically, and are generally associated with poor prognosis in patients with solid cancers [32]. Neutrophils may activate endothelium and parenchymal cells to enhance circulating tumor cell adhesion for distant metastasis [33]. Neutrophilia is an inflammatory response that inhibits the immune system by suppressing the cytolytic activity of immune cells such as lymphocytes, activated $T$ cells, and natural killer cells $[34,35]$. Secondly, platelets may act as protective "cloaks" for circulating tumor cells (CTCs), shielding them from immune destruction. Platelet and endothelial cell adhesion proteins may also facilitate metastasis by augmenting tumor cell extravasation [36]. Thirdly, the importance of lymphocytes has been highlighted in several studies in which high TILs (tumor-infiltrating lymphocytes) has been associated with better response to cytotoxic treatment and prognosis in cancer patients $[37,38]$. Lymphocytes can also secrete several cytokines, such as IFN- $\gamma$ and TNF-a, to block tumor growth and improve the prognosis of cancer patients [39]. According to the above theories, SII should be a more objective marker that reflects the balance between host inflammatory and immune response status than all the other systemic inflammation indices such as NLR, PLR and MLR.

Our study had several limitations. First, our study may be limited by selection biases due to its single-centre, retrospective nature. Second, although our results showed that SII was an independent predictor of ESCC prognosis, the sensitivity and specificity of SII were not very high. Third, although our findings were in line with previous observations, it was not easy to verify our conclusions in another independent cohort due to the lack of standardized cutoff value for SII. The cutoff values for SII were different among these similar studies [21, 22, 40, 41]. So, we need perspective studies to find a proper cutoff value. Thus, multi-center collaborative prospective studies are warranted to confirm these preliminary results.

\section{Conclusions}

The SII is an independent prognostic marker in patients with surgically resected ESCC, and the SII is superior to NLR, PLR and MLR in terms of prognostic ability. Moreover, SII retained prognostic significance in stage I-II ESCC subgroup (OS, DFS) and stage III ESCC subgroup (DFS).

\section{Abbreviations}

ESCC (esophageal squamous cell carcinoma); EAC (esophageal adenocarcinoma); OS (overall survival); CI (confidence interval); HR (hazard ratio); SII (immune-inflammation index); NLR (neutrophil lymphocyte ratio); PLR (platelet lymphocyte ratio); MLR (monocyte lymphocyte ratio); CBC (complete blood cell); ROC (receiver operated characteristics); AUC (area under the curve); CTCs (circulating tumor cells); TILs (tumor-infiltrating lymphocytes).

\section{Acknowledgements}

This study was supported by the National Key R\&D Program of China (2016YFC1303201, 2018YFC1312105), Beijing Nova Program (Z181100006218032), the CAMS Initiative for Innovative Medicine (2017-I2M-1-005), and the Non-profit Central Research Institute Fund of Chinese Academy of Medical Sciences (2017PT32001, PY2018B02).

\section{Competing Interests}

The authors have declared that no competing interest exists. 


\section{References}

1. Li N, Dhar SS, Chen TY, Kan PY, Wei Y, Kim JH, et al. JARID1D Is a Suppressor and Prognostic Marker of Prostate Cancer Invasion and Metastasis. Cancer Res. 2016; 76: 831-43.

2. Mao YS, He J, Cheng GY. [Current status of surgical management of esophageal cancer in China and the future strategy]. Zhonghua zhong liu za zhi [Chinese journal of oncology]. 2010; 32: 401-4.

3. Enzinger PC, Mayer RJ. Esophageal cancer. The New England journal of medicine. 2003; 349: 2241-52.

4. Tachibana M, Kinugasa S, Hirahara N, Yoshimura H. Lymph node classification of esophageal squamous cell carcinoma and adenocarcinoma. European journal of cardio-thoracic surgery : official journal of the European Association for Cardio-thoracic Surgery. 2008; 34: 427-31.

5. Crusz SM, Balkwill FR. Inflammation and cancer: advances and new agents. Nature reviews Clinical oncology. 2015; 12: 584-96.

6. Singel KL, Segal BH. Neutrophils in the tumor microenvironment: trying to heal the wound that cannot heal. Immunological reviews. 2016; 273: 329-43.

7. Franco AT, Corken A, Ware J. Platelets at the interface of thrombosis, inflammation, and cancer. Blood. 2015; 126: 582-8.

8. Dunn GP, Old LJ, Schreiber RD. The immunobiology of cancer immunosurveillance and immunoediting. Immunity. 2004; 21: 137-48.

9. Shao Y, Ning Z, Chen J, Geng Y, Gu W, Huang J, et al. Prognostic nomogram integrated systemic inflammation score for patients with esophageal squamouscell carcinoma undergoing radical esophagectomy. Scientific reports. 2015; 5: 18811.

10. Zhu Y, Li M, Bo C, Liu X, Zhang J, Li Z, et al. Prognostic significance of the lymphocyte-to-monocyte ratio and the tumor-infiltrating lymphocyte to tumor-associated macrophage ratio in patients with stage T3NOM0 esophageal squamous cell carcinoma. Cancer Immunology, Immunotherapy. 2017; 66: 343-54.

11. Feng J-F, Huang Y, Liu J-S. Combination of neutrophil lymphocyte ratio and platelet lymphocyte ratio is a useful predictor of postoperative survival in patients with esophageal squamous cell carcinoma. OncoTargets and therapy. 2013; 6: 1605.

12. Liu J-S, Huang Y, Yang X, Feng J-F. A nomogram to predict prognostic values of various inflammatory biomarkers in patients with esophageal squamous cell carcinoma. American journal of cancer research. 2015; 5: 2180.

13. Chen J-H, Zhai E-T, Yuan Y-J, Wu K-M, Xu J-B, Peng J-J, et al. Systemic immune-inflammation index for predicting prognosis of colorectal cancer. World journal of gastroenterology. 2017; 23: 6261.

14. Tomita M, Ayabe T, Maeda R, Nakamura K. Systemic Immune-inflammation Index Predicts Survival of Patients After Curative Resection for Non-small Cell Lung Cancer. in vivo. 2018; 32: 663-7.

15. Hu B, Yang X-R, Xu Y, Sun Y-F, Sun C, Guo W, et al. Systemic immune-inflammation index predicts prognosis of patients after curative resection for hepatocellular carcinoma. Clinical Cancer Research. 2014.

16. Hong $\mathrm{X}$, Cui B, Wang $\mathrm{M}$, Yang Z, Wang L, Xu Q. Systemic immune-inflammation index, based on platelet counts and neutrophil-lymphocyte ratio, is useful for predicting prognosis in small cell lung cancer. The Tohoku journal of experimental medicine. 2015; 236: 297-304.

17. Edge SB, Compton CC. The American Joint Committee on Cancer: the 7th edition of the AJCC cancer staging manual and the future of TNM. Annals of surgical oncology. 2010; 17: 1471-4.

18. Mantovani A, Allavena P, Sica A, Balkwill F. Cancer-related inflammation. Nature. 2008; 454: 436-44

19. Elinav E, Nowarski $R$, Thaiss CA, Hu B, Jin $C$, Flavell RA. Inflammation-induced cancer: crosstalk between tumours, immune cells and microorganisms. Nature reviews Cancer. 2013; 13: 759-71.

20. Trinchieri G. Cancer and inflammation: an old intuition with rapidly evolving new concepts. Annual review of immunology. 2012; 30: 677-706.

21. Feng J-F, Chen S, Yang X. Systemic immune-inflammation index (SII) is a useful prognostic indicator for patients with squamous cell carcinoma of the esophagus. Medicine. 2017; 96

22. Wang L, Wang C, Wang J, Huang X, Cheng Y. A novel systemic immune-inflammation index predicts survival and quality of life of patients after curative resection for esophageal squamous cell carcinoma. Journal of cancer research and clinical oncology. 2017; 143: 2077-86.

23. Hirahara N, Matsubara T, Mizota Y, Ishibashi S, Tajima Y. Prognostic value of preoperative inflammatory response biomarkers in patients with esophageal cancer who undergo a curative thoracoscopic esophagectomy. BMC surgery. 2016; 16: 66

24. Feng J-F, Huang Y, Chen Q-X. Preoperative platelet lymphocyte ratio (PLR) is superior to neutrophil lymphocyte ratio (NLR) as a predictive factor in patients with esophageal squamous cell carcinoma. World journal of surgical oncology. 2014; 12: 58.

25. Nakamura K, Yoshida N, Baba Y, Kosumi K, Uchihara T, Kiyozumi Y, et al. Elevated preoperative neutrophil-to-lymphocytes ratio predicts poor prognosis after esophagectomy in $\mathrm{T} 1$ esophageal cancer. International journal of clinical oncology. 2017; 22: 469-75.

26. Yutong $\mathrm{H}$, Xiaoli $\mathrm{X}$, Shumei $\mathrm{L}$, Shan $\mathrm{S}$, Di $\mathrm{L}$, Baoen $\mathrm{S}$. Increased neutrophil-lymphocyte ratio is a poor prognostic factor in patients with esophageal cancer in a high incidence area in China. Archives of medical research. 2015; 46: 557-63.
27. Hirahara N, Matsubara T, Kawahara D, Nakada S, Ishibashi S, Tajima Y. Prognostic significance of preoperative inflammatory response biomarkers in patients undergoing curative thoracoscopic esophagectomy for esophageal squamous cell carcinoma. European Journal of Surgical Oncology (EJSO). 2017; 43: 493-501.

28. Zhao Q-T, Zhang X-P, Zhang H, Duan G-C. Prognostic role of platelet to lymphocyte ratio in esophageal cancer: A meta-analysis. Oncotarget. 2017; 8: 112085.

29. Fan L, Wang R, Chi C, Cai W, Zhang Y, Qian H, et al. Systemic immune-inflammation index predicts the combined clinical outcome after sequential therapy with abiraterone and docetaxel for metastatic castration-resistant prostate cancer patients. The Prostate. 2018; 78: 250-6.

30. Lolli C, Basso U, Derosa L, Scarpi E, Sava T, Santoni M, et al. Systemic immune-inflammation index predicts the clinical outcome in patients with metastatic renal cell cancer treated with sunitinib. Oncotarget. 2016; 7: 54564.

31. Wang K, Diao F, Ye Z, Zhang X, Zhai E, Ren H, et al. Prognostic value of systemic immune-inflammation index in patients with gastric cancer. Chinese journal of cancer. 2017; 36: 75 .

32. Coffelt SB, Wellenstein MD, de Visser KE. Neutrophils in cancer: neutral no more. Nature Reviews Cancer. 2016; 16: 431

33. De Larco JE, Wuertz BR, Furcht LT. The potential role of neutrophils in promoting the metastatic phenotype of tumors releasing interleukin-8. Clinical Cancer Research. 2004; 10: 4895-900.

34. Petrie $\mathrm{H}$, Klassen $\mathrm{L}$, Kay $\mathrm{H}$. Inhibition of human cytotoxic $\mathrm{T}$ lymphocyte activity in vitro by autologous peripheral blood granulocytes. The Journal of Immunology. 1985; 134: 230-4.

35. El-Hag A, Clark R. Immunosuppression by activated human neutrophils. Dependence on the myeloperoxidase system. The Journal of Immunology. 1987; 139: 2406-13

36. Stanger BZ, Kahn ML. Platelets and tumor cells: a new form of border control. Cancer Cell. 2013; 24: 9-11.

37. Gooden MJ, de Bock GH, Leffers N, Daemen T, Nijman HW. The prognostic influence of tumour-infiltrating lymphocytes in cancer: a systematic review with meta-analysis. British journal of cancer. 2011; 105: 93.

38. Jia Q, Yang Y, Wan Y. Tumor-infiltrating memory T-lymphocytes for prognostic prediction in cancer patients: a meta-analysis. International journal of clinical and experimental medicine. 2015; 8: 1803.

39. Ferrone C, Dranoff G. Dual roles for immunity in gastrointestinal cancers. Journal of Clinical Oncology. 2010; 28: 4045.

40. Zhang H, Shang X, Ren P, Gong L, Ahmed A, Ma Z, et al. The predictive value of a preoperative systemic immune-inflammation index and prognostic nutritional index in patients with esophageal squamous cell carcinoma. Journal of Cellular Physiology. 2019; 234: 1794-802.

41. Geng Y, Shao Y, Zhu D, Zheng X, Zhou Q, Zhou W, et al. Systemic immune-inflammation index predicts prognosis of patients with esophageal squamous cell carcinoma: a propensity score-matched analysis. Scientific reports. 2016; 6: 39482 